\title{
Characterization of the Scattering and Absorption of Colored Zein Colloids in Optically Dense Dispersions
}

\author{
F. Y. de Boer, ${ }^{\dagger}$ R. J. A. van Dijk-Moes, ${ }^{\dagger}$ A. Imhof, ${ }^{*}{ }^{\dagger}$ a and K. P. Velikov ${ }^{\dagger, \ddagger}, \S$ \\ ${ }^{\dagger}$ Soft Condensed Matter \& Biophysics, Debye Institute for Nanomaterials Science, Utrecht University, Princetonplein 1, 3584 CC \\ Utrecht, The Netherlands \\ ${ }^{\ddagger}$ Unilever R\&D Vlaardingen, Olivier van Noortlaan 120, 3133 AT Vlaardingen, The Netherlands \\ ${ }^{\S}$ Institute of Physics, University of Amsterdam, Science Park 904, 1098 XH Amsterdam, The Netherlands
}

Supporting Information

ABSTRACT: In this research, we model the color of optically dense colloidal dispersions of dyed and undyed zein particles using results from multiple light scattering theory. These particles, as well as monodisperse silica colloids, were synthesized and characterized to obtain particle properties such as particle size, particle size distribution, refractive index, and absorption spectrum of the dye. This information was used to model the diffuse transmission of concentrated particle dispersions, which was measured using a specially designed variable path length quartz glass cuvette. For the nonabsorbing silica dispersions, a transport mean-free
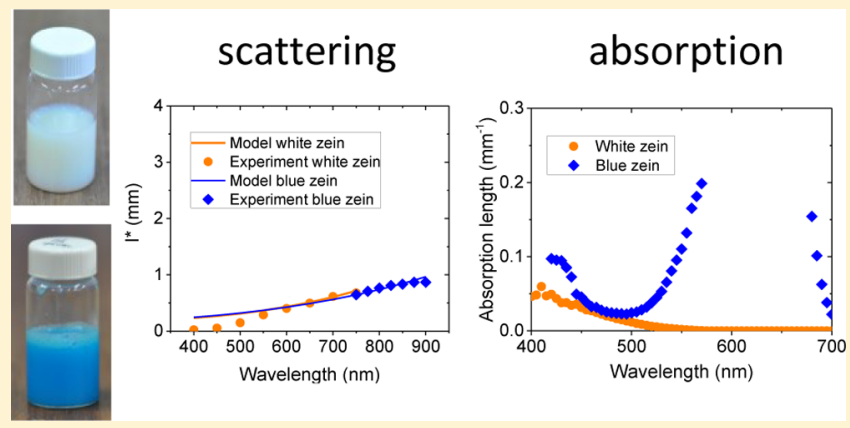
path throughout the visible range was obtained. Results showed a difference of less than $5 \%$ from the values calculated with a multiple scattering model using the single-particle properties as an input. For undyed zein particles, which are off-white, the deviation between the model and the experiment was about $30 \%$ because of slight absorption at wavelengths below $550 \mathrm{~nm}$ but $<7 \%$ at higher wavelengths. From these results, it was concluded that the model correctly describes diffuse transmission and that the measurements are sensitive to absorption. Finally, this method was applied to dispersions of dyed zein particles. Here, the transport mean-free path was first determined for wavelengths at which there is no absorption, which agreed with the theory better than $4 \%$. The modeled transport mean-free path was then used to extract the reciprocal absorption mean-free path in the remaining parts of the visible spectrum, and a reasonable agreement with the absorption spectrum of the dye solution was obtained.

\section{INTRODUCTION}

In the observation of color, the interaction of a material with light, scattering, and absorption play important roles. In colloids, this interaction is important for understanding their appearance. Colloids are particles dispersed in a medium that have at least in one direction a dimension roughly between 1 $\mathrm{nm}$ and $\mu \mathrm{m}$ or systems with discontinuities at distances of this order. ${ }^{1}$ These particles are found in everyday life, where they are, for example, applied in food industry, ${ }^{2}$ pharmaceuticals, ${ }^{3}$ inks, ${ }^{4}$ and coatings. ${ }^{5}$ Because many colloidal particles interact strongly with visible light, they have a color when observed. The contributing factors are their hierarchical order and scattering (structural colors), ${ }^{6,7}$ refractive index (RI) differences with the medium, their particle shape and size, ${ }^{8-10}$ absorption, and combinations of these.

Nowadays, it becomes clear that consumers prefer to buy products that contain natural and bio-based materials. A lot of research is being carried out toward this bio-based and natural trend, which is ranging from polymers and additives ${ }^{11,12}$ to foods and functional ingredients. ${ }^{13-15}$ A natural, bio-based class of materials to synthesize colloidal particles from is the class of proteins. Proteins have many advantageous properties, such as renewable, biodegradable, and often edible and soluble in food-safe solvents such as water-ethanol mixtures. ${ }^{16-18}$ These are the properties that make proteins suitable for many applications. For example, cellulose fibers ${ }^{19}$ were found to have enhanced light-scattering properties. Other studies showed that dried cellulose films may exhibit tunable optical properties $^{20,21}$ or stimuli-responsive optical properties. ${ }^{22}$ In our work, we have chosen to work with zein, a protein from corn, which has a GRAS (generally recognized as safe) status. The application areas for zein colloidal particles can be found in triggered-release mechanisms for drug delivery ${ }^{23}$ and also as a color carrier in food products, ${ }^{24}$ on which we focus in this study.

Because of the importance of color in everyday life and the widespread implementation of colloidal particles in a wide range of products, it would be beneficial if there were a simple way to predict optical properties from information about the constituent materials. This information consists primarily of

Received: May 9, 2019

Revised: August 23, 2019

Published: August 28, 2019 
the particle size and size distribution, the refractive indices of the medium and the particulate material, and the absorption spectrum of the colorant used. Different approaches to achieve this have been presented in literature already. ${ }^{25}$ The examples include full wave optical simulations suited for nanocomposites with extremely large filling fractions, ${ }^{26}$ density functional theory modeling for nanodiamond particles in a lattice structure, $^{27}$ and total differential effective medium theory for polymer nanocomposites, ${ }^{28}$ all of which are solid materials. Often, these are theoretical studies and mostly not compared to experimental results. Another approach, which was also applied on dispersions, is the Kubelka-Munk theory which simplifies the description of diffuse reflection from a layer in terms of two parameters, related to scattering and absorption, of a continuous medium. ${ }^{29-31}$ A drawback of the KubelkaMunk theory is that a direct connection with the optical properties of the individual particles is lost. Such a connection is retained by multiple light scattering (or photon diffusion) theory at the mild cost of moderately increased mathematical complexity. In this theory, diffusive light transport is quantified by the transport $\left(l^{*}\right)$ and absorption $\left(l_{\mathrm{a}}\right)$ mean-free paths, describing the effects of scattering and absorption. This theory has been highly successful in describing diffuse transmission and reflection of a wide variety of strongly scattering materials $^{32-36}$ and living tissues. ${ }^{37}$ Moreover, using diffusing wave spectroscopy particle dynamics in scattering, absorbing dispersions can be studied. ${ }^{38}$ A limitation of photon diffusion theory is that it fails for strongly absorbing samples. ${ }^{39}$ In this paper, we apply diffusion theory to polydisperse colloidal dispersions of absorbing (dyed) protein particles. Specifically, we attempt to relate the measured optical properties of the individual particles to the measured total transmission of opaque dispersions of these particles.

In this study, first the optical properties of nonabsorbing and absorbing colloidal protein particle dispersions were determined experimentally. Second, the transport and absorption mean-free paths were determined from total transmission measurements on optically dense dispersions. The mean-free paths were also calculated from Mie theory and multiple scattering theory and compared with the experimental data. To enable a comparison without adjustable parameters, information on particle shapes, sizes, size distributions, refractive indices, volume fractions, and the absorption spectrum of the colorant is necessary. To measure the total transmittance at a wide enough range of path lengths of the sample, a quartz glass cuvette with a variable path length was designed. To verify the setup, nonabsorbing, monodisperse silica particles were synthesized and measured using the newly designed setup. Following this, the experimental results of these silica particles were compared to the theory. When the setup was found to function as intended, this process was repeated for freshly synthesized white zein particles. Finally, the experiments and theory for absorbing blue zein particles were compared, resulting in a delimitation of the range of validity of the multiple scattering theory.

\section{EXPERIMENTAL SECTION}

Hydrochloric acid ( $\mathrm{HCl}$, Sigma), tetraethyl orthosilicate (TEOS, 99\%, Sigma), L-arginine (98\%, Sigma), Patent Blue V sodium salt (Sigma), dimethylsulfoxide (99.99\%, Sigma), 1-pentanol (99\%, Sigma), and absolute ethanol (Merck) were used as received. Zein was purchased from Flo Chemical Corporation (Zein F4000C-FG, lot nr.
F40006021C2), and water was purified using a Millipore Direct-Q purification system.

Particle Synthesis. Three types of particles were synthesized for different purposes. First, silica particles were synthesized as monodisperse and nonabsorbing particles to verify the performance of the transmittance setup and to test the theory. Then, white zein colloidal particles were synthesized to check whether these particles are also nonabsorbing and to test the sensitivity to polydisperse particles. Finally, the zein particles containing a blue dye were synthesized to measure their scattering and absorption.

Silica Synthesis. Silica particles were synthesized using the method of Shahabi et al. ${ }^{40}$ First, silica cores were prepared, which were grown into larger particles in a secondary step. L-Arginine (183 $\mathrm{mg}$ ) was dissolved in $169 \mathrm{~mL}$ of water in a base-bath-cleaned $500 \mathrm{~mL}$ one-neck flask. The mixture was heated to $70{ }^{\circ} \mathrm{C}$ and stirred slowly $(200 \mathrm{rpm})$ on a hot plate. After $1 \mathrm{~h}, 11.2 \mathrm{~mL}$ of TEOS was added slowly via the wall. The resulting mixture was stirred for 1 day to complete the core synthesis; this is known as the core dispersion.

The cores were grown into larger particles in two synthesis steps. In the first step, $163 \mathrm{mg}$ of L-arginine was dissolved in $152 \mathrm{~mL}$ of water, and then $19.3 \mathrm{~mL}$ of the core dispersion was added. The mixture was heated to $70{ }^{\circ} \mathrm{C}$ and stirred slowly $(200 \mathrm{rpm})$. After $1 \mathrm{~h}, 11.2 \mathrm{~mL}$ of TEOS was added slowly via the wall. The resulting dispersion was stirred for 1 day to complete the first growing step. In the second growing step, $162 \mathrm{mg}$ of $\mathrm{L}$-arginine was dissolved in $152 \mathrm{~mL}$ of water. Then, $19.3 \mathrm{~mL}$ of the particle dispersion from the first growth step was added. The mixture was again heated to $70{ }^{\circ} \mathrm{C}$ and stirred slowly $(200 \mathrm{rpm})$, and again after $1 \mathrm{~h}, 11.2 \mathrm{~mL}$ of TEOS was added slowly via the wall. The resulting dispersion was stirred for 1 day to complete the synthesis. Now, the particles are at their target size and concentration.

White Zein Particles. White zein particles were synthesized using the following procedure. ${ }^{41}$ Prior to particle synthesis, most of the removable colored impurities were extracted from the zein powder by washing in ethanol. Then, a stock solution was made in aqueous ethanol (85 wt \%), and $10 \mathrm{~mL}$ of stock solution was rapidly added to a beaker with water $(120 \mathrm{~mL})$ while stirring $(280 \mathrm{rpm})$. A 2 day dialysis of the resulting dispersion against water adjusted to a $\mathrm{pH}$ of 4 with $\mathrm{HCl}$ was used to completely remove the ethanol; during dialysis, the medium was replaced four times. The resulting dispersion was centrifuged for $30 \mathrm{~min}$ at $222 \mathrm{rcf}$ to remove possible large aggregates. Finally, the samples were stored in a fridge at $5{ }^{\circ} \mathrm{C}$ for a maximum of 2 weeks before measurement. The dry weight of the zein stock solutions and colloidal dispersions was determined by weighing an amount of the solution or dispersion, letting the liquid evaporate at $120{ }^{\circ} \mathrm{C}$ for $2 \mathrm{~h}$, and then measuring the dry weight. Prior to total transmission measurements, the zein particles were concentrated using a centrifugal concentrator (Vivaspin 20, pore size $100000 \mathrm{Da}$, Sartorius) at a pressure of 3 bar without centrifugation.

Blue Zein Particles. The blue zein particles were synthesized using a coprecipitation method. ${ }^{14,24}$ Before particle synthesis, the zein was purified by washing in ethanol, and a stock solution was prepared in 85 wt \% aqueous ethanol. Patent Blue V sodium salt was dissolved in the zein solution at a dye-to-zein ratio of 0.0025 prior to particle synthesis. To synthesize particles, $10 \mathrm{~mL}$ of the colored zein solution was quickly added to a beaker with water $(120 \mathrm{~mL})$ while stirring $(280 \mathrm{rpm})$. Directly after the synthesis, the encapsulation efficiency was determined. A 3 day dialysis of the resulting dispersion against water adjusted to a $\mathrm{pH}$ of 4 with $\mathrm{HCl}$ was performed to remove the remaining ethanol and free colorant. During dialysis, the dispersion was kept in the dark to prevent possible color changes. During this dialysis, the medium was replaced four times. The resulting colloidal dispersion was then centrifuged for $30 \mathrm{~min}$ at $222 \mathrm{rcf}$ to remove possible large aggregates. Finally, the samples were stored in a fridge at $5{ }^{\circ} \mathrm{C}$ for a maximum of 2 weeks before measurement. Prior to total transmission measurements, the zein particles were concentrated using a centrifugal concentrator (Vivaspin 20, pore size $100000 \mathrm{Da}$, Sartorius) at a pressure of 3 bar without centrifugation.

Particle Shape, Size, and Size Distribution. Transmission electron microscopy (TEM, Technai operating at $100 \mathrm{kV}$ equipped 
with a tungsten filament) was used to determine the particle sizes of the cores and the results of the two growing steps from the silica synthesis. Samples for analysis were prepared by first sonicating the silica dispersions for $1 \mathrm{~min}$ to prevent aggregation of the particles on the TEM grid. Then, the silica dispersion was drop-cast onto carboncoated copper TEM grids at room temperature and the liquid was evaporated in air, leaving the silica particles on the grid.

Scanning electron microscopy (SEM, Nova Nanolab, FEI) was used for determining the particle shape of the samples prepared from zein. To minimize the changes in particle size and shape, the samples were freeze-dried overnight using a lyophilizer (Virtis). The dry powder, which was loosely attached to the lyophilizing bottle, was collected and stored in a desiccator until SEM analysis. Prior to SEM analysis, a platinum layer of about $4 \mathrm{~nm}$ was sputtered onto these samples to prevent charging.

The size distributions and particle sizes were also determined by dynamic light scattering (DLS) using a Zetasizer Nano ZS series (Malvern Instruments). In DLS, a CONTIN analysis was used to determine the size distributions. Prior to the DLS measurements, the samples were diluted with water adjusted to $\mathrm{pH} 4$ to a suitable concentration in order to prevent multiple scattering.

Dye Encapsulation Efficiency. Directly after the coprecipitation in the blue particle synthesis, a sample of the dispersion was filtered using a Vivaspin tube (Sartorius, equipped with a 100000 MWCO membrane) using an applied pressure of 4 bar to separate the particles from the medium. The encapsulation efficiency or adsorption was determined as follows

$$
\mathrm{EE}=\frac{c_{\mathrm{t}}-c_{\mathrm{m}}}{c_{\mathrm{t}}} 100 \%
$$

Here, EE is the encapsulation efficiency or adsorption, $c_{\mathrm{t}}$ is the concentration of dye added to the synthesis, and $c_{\mathrm{m}}$ is the concentration of free dye in the medium. From this calculation, the total concentration of dye inside the particles, $c_{\mathrm{p}}$, can be calculated from $c_{\mathrm{p}}=c_{\mathrm{t}}-c_{\mathrm{m}}$. UV-vis spectroscopy (HP 8953A spectrophotometer) was used to determine the concentration of free dye in the medium at a wavelength of $638 \mathrm{~nm}$. The concentration was calculated by comparison with an appropriate calibration curve using the same medium as the particle dispersions.

Optical Properties of Concentrated Dispersions. The transport mean-free path $\left(l^{*}\right)$ and reciprocal absorption mean-free path $(\alpha)$ were experimentally determined via total transmittance spectroscopy, which was performed on an in-house-built setup (Figure 1). The concentrated samples were measured at wavelengths ranging from 400 to $900 \mathrm{~nm}$ using a specially designed quartz glass cuvette with a variable path length, with an accuracy of $<0.05 \mathrm{~mm}$ after initial calibration because of the use of an optical linear stage (Figure 1),

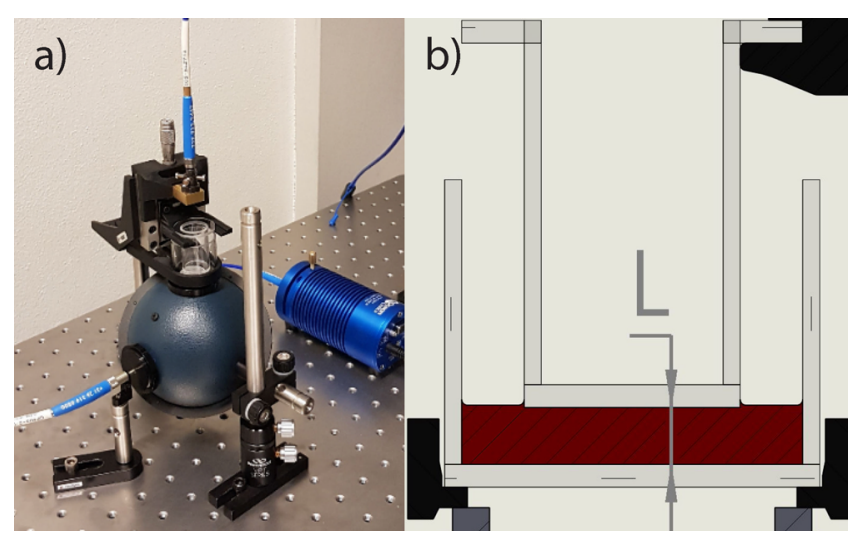

Figure 1. (a) In-house-built setup for the variable path length cell, including a schematic cross section of the designed quartz cuvettes (b), 3D printed holder incorporating a linear stage and a separate, stationary holder for the fiber collimator, and an integrating sphere and light source that was used for the measurements.
Newport M-UMR5.16 with BM11.16. The structural parts consist of a $90^{\circ}$ bracket, Newport EQ50-E, and custom-designed 3D printed parts printed in PLA on an Ultimaker2 FDM 3D printer. The inner and outer quartz glass cylinders had diameters of 17 and $30 \mathrm{~mm}$, respectively. Light from a Tungsten Halogen light source (HL-2000FHSA-LL, Ocean Optics) was sent vertically down via a multimode optical fiber through the pair of circular optical flats that form the bottom of the cylinders. Their separation varied in the range of 0.5$10 \mathrm{~mm}$ by raising and lowering the inner cylinder via the optical linear stage. Transmitted light was collected by a $15 \mathrm{~cm}$ diameter integrating sphere (barium sulfate coated, Labsphere) with an entrance opening of $18 \mathrm{~mm}$. The sphere had a detection port of $3 \mathrm{~mm}$, at which light was collected by a multimode optical fiber and coupled into a spectrometer (HR4000, Ocean Optics). The modular design of the setup enables quick and easy modifications for cleaning purposes and alternative configurations if required.

\section{RESULTS AND DISCUSSION}

Particle Characterization. The particle growth of the silica particles was followed by TEM, see Supporting Information Figures S1 and 2a. First, seeds were grown with a size of about $26 \mathrm{~nm}$ (measured from TEM images); then, the particles were grown to about $55 \mathrm{~nm}$ and finally to their final size of $128 \mathrm{~nm}$. These silica particles are spherical and nonabsorbing and can therefore be used to verify the setup and theory.

The spherical shape of the zein particles (white and blue) was verified by SEM, see Figure $2 \mathrm{~b}, \mathrm{c}$. Compared to the silica particles, these zein particles are very polydisperse, which has been observed before for this type of particles in literature. ${ }^{42,43}$ There does not seem to be a difference in shape between white zein particles and blue zein particles, as they both appear spherical.

To exclude the differences between sample preparations and microscopy techniques, DLS was used to determine the size and size distribution of the different particles. This resulted in particle sizes of silica: $140 \pm 24 \mathrm{~nm}$, white zein: $149 \pm 60 \mathrm{~nm}$, and blue zein: $156 \pm 77 \mathrm{~nm}$. Figure $2 \mathrm{~d}-\mathrm{f}$ presents the size distribution data obtained with DLS, which are used in the model for the calculation of transport mean-free path $\left(l^{*}\right)$.

The encapsulation efficiency of the blue zein particles was determined using eq 1 and found to be $99.9 \%$, which is in agreement with previous experiments. ${ }^{14}$

The RI of zein dispersed in water was determined from extinction measurements in our previous study. ${ }^{41} \mathrm{~A}$ value of $1.49 \pm 0.01$ was found to describe the extinction of a large number of similarly prepared, polydisperse samples in the wavelength range of $450-700 \mathrm{~nm}$; this value is used in this study for both the white and blue zein particles, regardless of the wavelength. The RI of silica was determined by RI matching as explained in Supporting Information S2 and found to be 1.453 , which is comparable to other values that can be found for silica particles in literature. ${ }^{44,45}$

Multiple Scattering of Optically Dense Dispersions. Nonabsorbing Dispersions. The case of multiple scattering of nonabsorbing polydisperse zein particles was described in our previous work. ${ }^{41}$ Here, the same phenomenon is described, now measured using the variable path length cell setup and using monodisperse silica to better compare the theory with experiments. Because the mean-free paths of our samples will turn out to be $0.2-2 \mathrm{~mm}$ (determined experimentally), the maximum sample path lengths will be made several times that length (up to $6 \mathrm{~mm}$ ). At the same time, the cell is wide enough $(30 \mathrm{~mm})$ to prevent any light leaking out through the side 

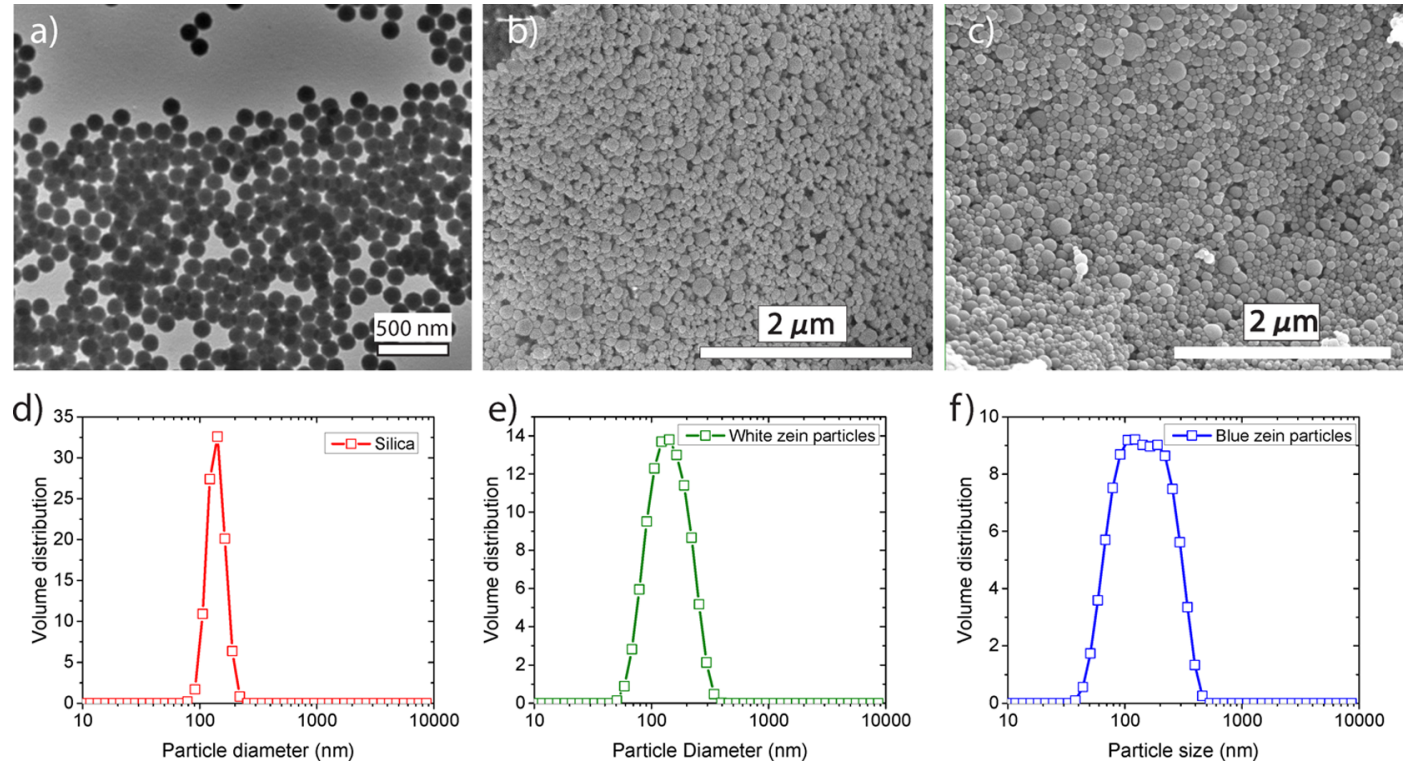

Figure 2. TEM image of silica particles after the final growth step (scale bar is $500 \mathrm{~nm}$ ) after which the particles were approximately $128 \mathrm{~nm}$ (a), which were used for further experiments. SEM images of white zein particles (b) and blue zein particles (c). After freeze-drying, the particles were found to be spherical, although both samples were very polydisperse. Size distribution (volume) data for silica particles (d), white zein particles (e), and blue zein particles (f). The size distribution data were obtained from DLS.

walls, even at the larger cell thicknesses. If these conditions were not met, the resulting light loss could masquerade as absorption. This improvement will allow us to include absorption into the model, as described in the next section. Only opaque dispersions are used, so light is scattered not once but multiple times before leaving the sample. With volume fractions of a few percent, these dispersions are dilute in the sense that particles interact only weakly. However, they are optically dense as no light is transmitted without scattering. The scattering strength is quantified by the transport mean-free path, $l^{*}$, which describes the distance over which the direction of propagation of light is randomized

$$
\frac{1}{l^{*}}=\frac{1-\langle\cos \theta\rangle}{l}
$$

In this equation, $l$ is the scattering mean-free path (the average distance between scattering events) and $\langle\cos \theta\rangle$ is the anisotropy factor, the average of the cosine of the scattering angle $\theta$, both of which can be calculated from Mie theory. ${ }^{46}$ In the absorption-free case, the transport mean-free path can be experimentally determined from the relation ${ }^{36}$ between the total transmittance $T$ and the path length of the cell, $L$ (eq 3 ), which shows that the total diffuse transmittance is low at small $l^{*}$. Here, most of the incident light is diffusely reflected

$$
T=\left(1-R_{\mathrm{s}}\right) \frac{1+z_{\mathrm{e}}}{\frac{L}{l^{*}}+2 z_{\mathrm{e}}}, \text { with } z_{\mathrm{e}}=\frac{2}{3} \frac{1+\bar{R}}{1-\bar{R}}
$$

In this equation, $\bar{R}$ is the polarization and angle-averaged internal diffuse reflectivity of the sample-quartz glass-air interface, $R_{\mathrm{s}}$ is the specular reflectivity of the incident light beam from the front face of the sample, and $z_{\mathrm{e}}$ is the extrapolation length ratio, which describes the boundary conditions of the diffuse intensity at the interfaces of the sample. $R_{\mathrm{s}}$ and $\bar{R}$ can be calculated via Fresnel's reflection coefficients as described by Vera and Durian ${ }^{47}$ from the RIs of the sample medium, container wall, and external medium (i.e., $1.330,1.458$, and 1.000 respectively, at $589 \mathrm{~nm}$ ), which leads to $R_{\mathrm{s}}=0.0367, \bar{R}=0.4452$, and $z_{\mathrm{e}}=1.7365$. Finally, $l^{*}$ is obtained from the slope of the linear fit of the $1 / T$ versus $L$ plot.

The experimentally obtained $l^{*}$ values are shown in Figure 3. For silica particles, this fit had a $R^{2}$ of 0.990 or higher for all

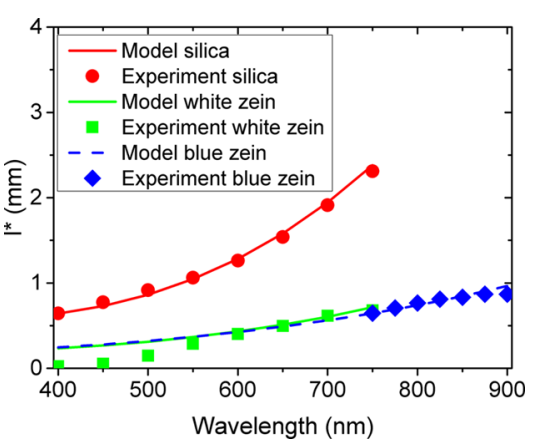

Figure 3. Transport mean-free path, $l^{*}$, found experimentally by total transmittance spectroscopy (points) and calculated from theory through eq 4 (lines) of a silica particle dispersion (particle diameter $140 \pm 24 \mathrm{~nm}$ and $\varphi=0.014$ ), a white zein particle dispersion (particle diameter $149 \pm 60 \mathrm{~nm}$ and $\varphi=0.024)$, and a blue zein particle dispersion (particle diameter $156 \pm 77 \mathrm{~nm}$ and $\varphi=0.025$ ). In the blue zein case, only data are shown at wavelengths where absorption is absent, and the theory is used to extrapolate to wavelengths where absorption cannot be neglected. In the white zein case, neglecting absorption below $550 \mathrm{~nm}$ underestimates $l^{*}$.

wavelengths (between 400 and $750 \mathrm{~nm}$ ), as shown in Supporting Information Figure S3. From this result, it can be concluded that the experimental setup works according to the expectations. The white zein particles give equally good fits at longer wavelengths but clearly deviate from a linear fit for wavelengths below $550 \mathrm{~nm}$ (see Supporting Information Figure S4). This could be a sign of absorption for these specific wavelengths, which will be considered in the next section. 

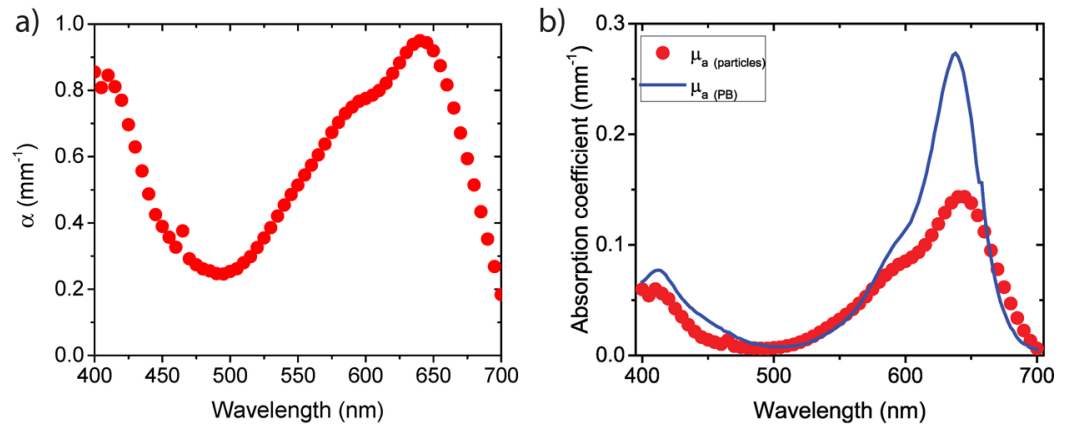

Figure 4. Reciprocal absorption mean-free path, $\alpha$, of the blue particle dispersion found experimentally by total transmittance spectroscopy (a) and calculated absorption coefficients using eq 7 compared to the absorption spectrum of Patent Blue V sodium salt in water (b).

As shown in our previous study, ${ }^{41}$ the transport mean-free path $l^{*}$ for a polydisperse sample is related to the particle properties as

$$
\begin{aligned}
& \left\langle\frac{Q_{\text {ext }}}{r}(1-\langle\cos \theta\rangle)\right\rangle \\
& \equiv \frac{\sum_{i} f_{i} r_{i}^{3} \frac{Q_{\text {ex }, i}}{r_{i}}\left(1-\left\langle\cos \theta_{i}\right\rangle\right)}{\sum_{i} f_{i} r_{i}^{3}} \\
& =\frac{1}{l^{*}} \frac{4}{3 \varphi}
\end{aligned}
$$

Here, $\varphi$ is the total volume of particles and $Q_{\text {ext, } i}=C_{\text {ext, } i} / r_{i}$ with $C_{\text {ext }, i}$ being the scattering cross section of a particle with size $r_{i}$. The summation accounts for the scattering contributions of particles of different sizes $r_{i}$, from a distribution $f_{i}$, which are calculated with Mie theory. The particle volume distribution $f_{i} r_{i}^{3}$ is taken from the size distribution data from DLS results of the corresponding samples (see Figure 2). Note that here the assumption is made that the scattering particles are uncorrelated, so that effects of the structure factor can be neglected. This is acceptable for the relatively low concentrations $(<3 \mathrm{wt} \%)$ used in this work. The Mie theory software MiePlot $^{48}$ was used for the calculation of $Q_{\text {ext }}$ and $\langle\cos \theta\rangle$. The results are included in Figure 3.

For both the silica and white zein particle dispersions, the agreement between the model and theory is very good, especially when considering that no adjustable parameters were used. Deviations between experimental results and theoretical calculations, as prescribed by eq 4 , are observed to be $\leq 5 \%$ for the silica particles. This is as expected because silica is nonabsorbing and will only scatter the light ${ }^{49}$ and also the silica particles are spherical and monodisperse, as observed in TEM and DLS measurements. Note that we have neglected RI dispersion, as calculations showed that a reasonable RI variation of 0.01 over the measured range would change the predicted $l^{*}$ by less than $9 \%$.

For white zein particle dispersions, deviations between experiments and theory are on average about $30 \%$, which is very similar to the result obtained before with only fixed path length cells. ${ }^{41}$ However, when looking just at wavelengths of $\geq 550 \mathrm{~nm}$, the agreement is better than $7 \%$, which is a large improvement compared to our previous work. It can also be observed that the values of the experimental results of white zein particles $\leq 550 \mathrm{~nm}$ are below the theoretical data line, which indicates that the samples transmit less light than expected based on their scattering cross sections. This is again an indication that these white zein particles still absorb some light at these wavelengths, resulting experimentally in very low values of $l^{*}$. The strong scattering of these samples increases the effective path length of transmitted light, strongly amplifying even low amounts of absorption. Therefore, we will now extend the theory to include absorption.

Absorbing Dispersions. For the case in which particles absorb and scatter light, the equations are in principle similar to the nonabsorbing case as was described before. The analytical solution of the diffusion equation in the presence of absorption is, for the case of collimated normal-incident light, known to be 39

$$
T=\left(1-R_{\mathrm{s}}\right) \frac{1}{\alpha z_{\mathrm{e}}} \frac{\sinh \left[\alpha\left(l^{*}+z_{\mathrm{e}}\right)\right] \sinh \left[\alpha z_{\mathrm{e}}\right]}{\sinh \alpha\left(L+2 z_{\mathrm{e}}\right)}
$$

Here, $\alpha$ is the reciprocal absorption mean-free path, $\alpha=1 / l_{\mathrm{a}}$. The absorption mean-free path is the distance it takes light to be absorbed to a fraction $1 / e$ while performing a random walk in the scattering sample. The extrapolation length, $z_{\mathrm{e}}$, is also modified in the absorbing case

$$
z_{\mathrm{e}}=\frac{1}{2 \alpha} \ln \left(\frac{1+\alpha z_{0}}{1-\alpha z_{0}}\right) \text {, with } z_{0}=\frac{2}{3} l * \frac{1+\bar{R}}{1-\bar{R}}
$$

It can be checked that eq 5 reduces to eq 3 in the limit of low absorption $\left(\alpha \ll 1 / l^{*}\right)$. A direct two-parameter fit of $l^{*}$ and $\alpha$ using eqs 5 and 6 unfortunately produced unreliable results because the two parameters mainly enter the equations as their product. Therefore, we determined $l^{*}$ first, after which $\alpha$ was fitted using eq 5 , as follows. Because eq 4 has proven to be a reliable model, we use it to obtain $l^{*}$ at wavelengths where absorption becomes important. Figure 3 shows the predictions of eq 4 and compares them with experimental results determined through eq 3 for wavelengths without absorption $(750-900 \mathrm{~nm})$. It is seen that the agreement is quite good (better than $4 \%$ ). The corresponding $1 / T$ versus $L$ plots are shown in Supporting Information Figure S5. The extrapolation is helped by the fact that the wavelength dependence of $l^{*}$ is weak. It has been shown that in highly polydisperse systems, the transport mean-free path is only weakly dependent on wavelength. ${ }^{39,50}$ This is also visible in the data shown in Figure 3 , in which silica is reasonably monodisperse and indeed wavelength-dependent. White zein particles, which are much more polydisperse, show much less wavelength dependency.

Then, $\alpha$ was obtained from a fit of $T$ versus $L$ to eq 5 in the absorbing region $400-700 \mathrm{~nm}$. The corresponding fits are shown in Supporting Information Figure S6. The results are presented in Figure 4a. As expected, the resulting spectrum is similar in shape to the absorption spectrum of patent blue in 

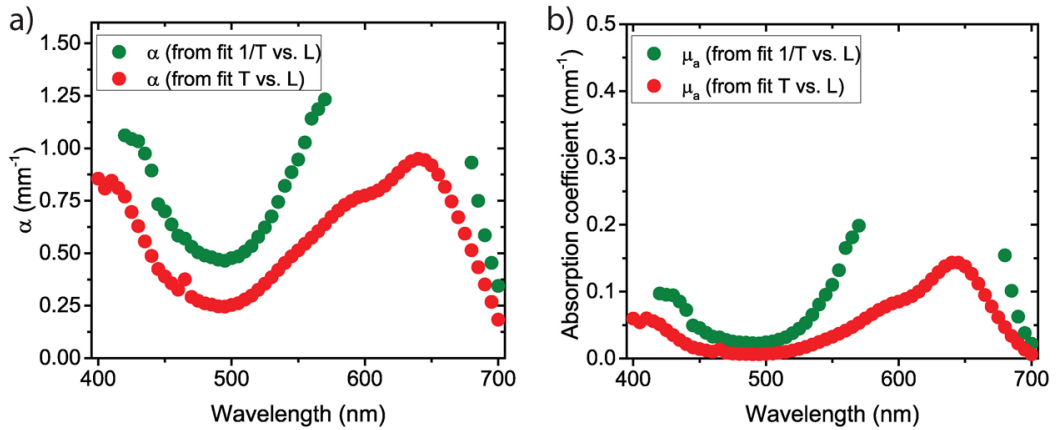

Figure 5. Reciprocal absorption mean-free path, $\alpha$, of the blue particle dispersion found experimentally by total transmittance spectroscopy (a) and calculated absorption coefficient using eq 7 (b) using fits from both $T$ vs $L$ and $1 / T$ vs $L$ plots. The values obtained from the $1 / T$ plots are quantitatively correct but cannot be determined at wavelengths with strong absorption.
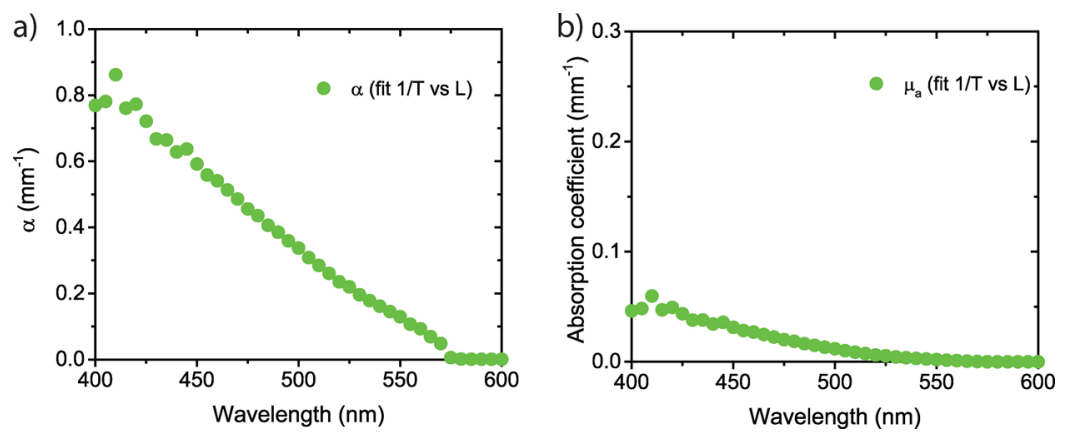

Figure 6. Reciprocal absorption mean-free path, $\alpha$, of the white zein particle dispersion of Figure 3 found experimentally by total transmittance spectroscopy and using fits from $1 / T$ vs $L$ plots (a) and corresponding calculated absorption coefficients using eq 7 (b).

water using the same dye concentration (i.e., without scattering, Figure $4 \mathrm{~b}$, blue line). The peaks and even the shoulder at $590 \mathrm{~nm}$ are well reflected in the measured spectrum. Because scattering is strongest at short wavelengths, the absorption peak at $410 \mathrm{~nm}$ is strongly enhanced compared to the peak at $640 \mathrm{~nm}$. To make a quantitative comparison between the absorption mean-free path $\alpha$ measured on the dyed zein dispersion and the absorption coefficient $\mu_{\mathrm{a}}=(1 / L)$ $\log T$ of the pure dye solution, we calculate the absorption coefficient of the dispersion using ${ }^{51}$

$$
\mu_{\mathrm{a}}=\frac{l^{*} \alpha^{2}}{3}
$$

Figure $4 \mathrm{~b}$ shows that the agreement with the two data sets is reasonable. However, the absorption peaks from the dye in water are considerably higher than for the particle dispersion. There also seems to be a small shift in peak position. This is a known phenomenon that can occur for Patent Blue V sodium salt when the dye is in a different medium or in a medium with a different $\mathrm{pH}^{52-54}$

On the other hand, the difference between data sets can be explained by the quality of the fits (Supporting Information Figure S6), which is not as good as would be desirable. A much-improved fit is obtained by plotting $1 / T$ versus $L$, see Supporting Information Figure S7. Although the fit is better at wavelength regions with low to moderate absorption, it was not possible to fit the wavelength region with strong absorption (570-680 $\mathrm{nm}$ ) using eqs 5 and 6 because of strong absorption of the sample for these wavelengths.

It has been shown by Leung et al. that eq 5 becomes unphysical for samples with very strong absorption compared to scattering. ${ }^{39}$ This is because the extrapolation length in the presence of absorption, $z_{\mathrm{e}}$, diverges if the absorption mean-free path, $l_{a}$, becomes similar to or smaller than the extrapolation length without absorption, $z_{0}$. Then, in eq 6 , when $1-\alpha z_{0}>1$, the logarithmic part of eq 5 becomes divergent and cannot be used anymore.

For the wavelengths for which it was possible to find a fit, $\alpha$ and the absorption coefficient were calculated (see Figure 5) and compared to the results from Figure 4. A clear difference in magnitude can be observed: the values found using the better fit are much higher. Figure $5 b$ suggests that the absorption peak is much higher and possibly more comparable to the absorption spectrum of the dye in water. However, because of strong absorption, the data are outside the range of applicability of diffusion theory.

When scattering particles also absorb, they should be described with a complex RI, the imaginary part of which is

$$
n^{\prime \prime}=\frac{\lambda}{4 \pi} \cdot \frac{\mu_{\mathrm{a}}}{\varphi}
$$

where $\lambda$ is the wavelength in vacuum. Because in our case all dye is concentrated in the particles, the factor $\mu_{\mathrm{a}} / \varphi$ is the absorption coefficient of the zein particles themselves. Including the complex RI in the calculations might change the values of the scattering cross section and anisotropy factor, invalidating our earlier analysis (which included only the real part of the RI). However, assuming a high value of $\mu_{\mathrm{a}}=1$ $\mathrm{mm}^{-1}$ at $630 \mathrm{~nm}$, we obtain $n^{\prime \prime}=0.002$. Using this value in a Mie calculation, we find that the scattering cross section and anisotropy factor change by only $0.1 \%$. We conclude, therefore, that the use of an imaginary part of the RI is not needed unless the particles are loaded with several times the amount of dye used in our experiments. 
Finally, we return to the white zein particles to quantify their suspected absorption at wavelengths below $550 \mathrm{~nm}$ (see Figure 3 ). For these particles, the absorption is not strong, and eq 5 should be valid over the whole wavelength spectrum.

We note that when $\alpha$ is obtained from $T$ versus $L$ plots (Supporting Information Figure S8) also here, the results were not optimal. Therefore, fits were obtained by calculating $\alpha$ from plotting $1 / T$ versus $L$, see Supporting Information Figure S9. Because this dispersion is low in absorption, it was possible to find a good fit for all wavelengths of interest. From these fits, the $\alpha$ and the absorption coefficients were determined, see Figure 6. Here, it is visible that there is indeed some absorption, mainly below $550 \mathrm{~nm}$ as was expected from the result in Figure 3.

When comparing the results from the white zein particles in Figure 6 to the blue zein particles in Figure 5, it looks like the reciprocal absorption mean-free path of the white zein particles is almost as high as for the dyed zein particles. When an unsuitable fit (fit of $T$ vs $L$ ) is used, this indeed appears to be the case. However, when a more suitable fit (fit $1 / T$ vs $L$ ) is used, it is not possible to reach higher values of $\alpha$ at absorbing wavelengths because the fitted equation does not allow for it (this explains why there is a gap in Figure 5 for the green dots). If it had been possible to fit these points, then the values of $\alpha$ would have been much higher for the blue particles than for the white particles.

\section{CONCLUSIONS}

In this study, colloidal particles were synthesized and characterized to obtain particle properties such as particle size, particle size distribution, RI, and absorption spectrum of the dye. This information was used to model the optical properties of these concentrated particle dispersions, which were experimentally measured using a specially designed variable path length quartz glass cuvette.

Nonabsorbing spherical and monodisperse silica particles were synthesized to verify the correct workings of the setup and to check the applicability of multiple scattering theory. The agreement between the experiment and theory, in terms of the transport mean-free path of these silica particles, was better than $5 \%$. The same experiment was performed on polydisperse white zein particles, which were slightly absorbing below wavelengths of $550 \mathrm{~nm}$. Again, good agreement between theory and experiments was obtained: better than $7 \%$ for wavelengths above $550 \mathrm{~nm}$. Deviations at shorter wavelengths were attributed to some absorption by the zein and are indicative of the sensitivity of the method to absorption. Subsequently, absorbing and polydisperse blue zein particles were measured using the setup. For these particles, the transport mean-free path was determined for wavelengths where absorption is negligible, which gave a deviation between theory and experiment of $\leq 4 \%$. The modeled transport meanfree path was then used to find the reciprocal absorption meanfree path and was compared to the absorption spectrum of the dye, which was found to have reasonable agreement. However, at the absorption peaks, the agreement was less good because of the fact that the absorption became stronger than the scattering. For a complete agreement, a theory other than diffusion theory needs to be used to describe light transport. The diffusion theory worked best for the slightly absorbing white zein dispersion.

These results show that it is possible to predict the scattering and absorption behavior of opaque dispersions using the RI, volume fraction, dye absorption spectrum, particle size, and size distribution as inputs. This work is a step forward when particles with specifically predesigned optical properties must be synthesized, which can be tuned toward a specific application.

\section{ASSOCIATED CONTENT}

\section{Supporting Information}

The Supporting Information is available free of charge on the ACS Publications website at DOI: 10.1021/acs.langmuir.9b01357.

All TEM images obtained for all steps in the silica synthesis; methods and results for the RI matching of silica particles; and fitting curves obtained by total transmission experiments (PDF)

\section{AUTHOR INFORMATION}

\section{Corresponding Author}

*E-mail: a.imhof@uu.nl.

ORCID $\odot$

F. Y. de Boer: 0000-0002-3163-3236

A. Imhof: 0000-0002-7445-1360

\section{Author Contributions}

This manuscript was written through contributions from all authors. All authors have given approval to the final version of the manuscript.

Notes

The authors declare no competing financial interest.

\section{ACKNOWLEDGMENTS}

The authors would like to thank Chris Schneijdenberg for his assistance in using SEM, Allard Mosk for useful discussions, Jantina Fokkema for her help with coding Mathematica, and Jim Veerkamp for his assistance in the design of the variable path length cell. This research was supported by the Dutch Technology Foundation STW (grant 13567), which is part of the Netherlands Organization for Scientific Research (NWO) and partly funded by the Ministry of Economic Affairs.

\section{REFERENCES}

(1) IUPAC. Compendium of Chemical Terminology Choice Reviews Online, 2013; Vol. 39 (08), pp 39-4583.

(2) Patel, A. R. Functional and Engineered Colloids from Edible Materials for Emerging Applications in Designing the Food of the Future. Adv. Funct. Mater. 2018, 4, 1806809.

(3) Bonacucina, G.; Cespi, M.; Misici-Falzi, M.; Palmieri, G. F. Colloidal Soft Matter as Drug Delivery System. J. Pharm. Sci. 2009, 98, 1-42.

(4) Rajan, K.; Roppolo, I.; Chiappone, A.; Bocchini, S.; Perrone, D.; Chiolerio, A. Silver nanoparticle ink technology: state of the art. Nanotechnol. Sci. Appl. 2016, 9, 1-13.

(5) Jiang, S.; Van Dyk, A.; Maurice, A.; Bohling, J.; Fasano, D.; Brownell, S. Design Colloidal Particle Morphology and Self-Assembly for Coating Applications. Chem. Soc. Rev. 2017, 46, 3792-3807.

(6) Xiao, M.; Li, Y.; Allen, M. C.; Deheyn, D. D.; Yue, X.; Zhao, J.; Gianneschi, N. C.; Shawkey, M. D.; Dhinojwala, A. Bio-Inspired Structural Colors Produced via Self-Assembly of Synthetic Melanin Nanoparticles. ACS Nano 2015, 9, 5454-5460.

(7) Vogel, N.; Utech, S.; England, G. T.; Shirman, T.; Phillips, K. R.; Koay, N.; Burgess, I. B.; Kolle, M.; Weitz, D. A.; Aizenberg, J. Color from Hierarchy: Diverse Optical Properties of Micron-Sized Spherical Colloidal Assemblies. Proc. Natl. Acad. Sci. U.S.A. 2015, 112, 1084510850. 
(8) Huang, T.; Xu, X.-H. N. Synthesis and Characterization of Tunable Rainbow Colored Colloidal Silver Nanoparticles Using Single-Nanoparticle Plasmonic Microscopy and Spectroscopy. J. Mater. Chem. 2010, 20, 9867-9876.

(9) Liz-Marzán, L. M. Nanometals. Mater. Today 2004, 7, 26-31.

(10) Turkevich, J.; Garton, G.; Stevenson, P. C. The Color of Colloidal Gold. J. Colloid Sci. 1954, 9, 26-35.

(11) Imam, S. H.; Bilbao-Sainz, C.; Chiou, B.-S.; Glenn, G. M.; Orts, W. J. Biobased Adhesives, Gums, Emulsions, and Binders: Current Trends and Future Prospects. J. Adhes. Sci. Technol. 2013, 27, 19721997.

(12) Vieira, M. G. A.; da Silva, M. A.; dos Santos, L. O.; Beppu, M. M. Natural-Based Plasticizers and Biopolymer Films: A Review. Eur. Polym. J. 2011, 47, 254-263.

(13) Joye, I. J.; Davidov-Pardo, G.; McClements, D. J. Nanotechnology for Increased Micronutrient Bioavailability. Trends Food Sci. Technol. 2014, 40, 168-182.

(14) de Boer, F. Y.; Imhof, A.; Velikov, K. P. Color-Tunable Particles through Affinity Interactions between Water-Insoluble Protein and Soluble Dyes. Colloids Surf., A 2019, 562, 154-160.

(15) de Boer, F. Y.; Imhof, A.; Velikov, K. P. Encapsulation of Colorants by Natural Polymers for Food Applications. Color. Technol. 2019, 135, 183-194.

(16) Nesterenko, A.; Alric, I.; Silvestre, F.; Durrieu, V. Vegetable Proteins in Microencapsulation: A Review of Recent Interventions and Their Effectiveness. Ind. Crops Prod. 2013, 42, 469-479.

(17) Hoogenkamp, H.; Kumagai, H.; Wanasundara, J. P. D. Rice Protein and Rice Protein Products. In Sustainable Protein Sources; Elsevier, 2017; pp 47-65.

(18) Rishi, S.; Munir, C. Zein: Industrial Protein from Corn. Ind. Crops Prod. 2001, 13, 171-192.

(19) Caixeiro, S.; Peruzzo, M.; Onelli, O. D.; Vignolini, S.; Sapienza, R. Disordered Cellulose-Based Nanostructures for Enhanced Light Scattering. ACS Appl. Mater. Interfaces 2017, 9, 7885-7890.

(20) Guidetti, G.; Atifi, S.; Vignolini, S.; Hamad, W. Y. Flexible Photonic Cellulose Nanocrystal Films. Adv. Mater. 2016, 28, 1004210047.

(21) Parker, R. M.; Guidetti, G.; Williams, C. A.; Zhao, T.; Narkevicius, A.; Vignolini, S.; Frka-Petesic, B. The Self-Assembly of Cellulose Nanocrystals: Hierarchical Design of Visual Appearance. Adv. Mater. 2018, 30, 1704477.

(22) Kose, O.; Tran, A.; Lewis, L.; Hamad, W. Y.; MacLachlan, M. J. Unwinding a Spiral of Cellulose Nanocrystals for Stimuli-Responsive Stretchable Optics. Nat. Commun. 2019, 10, 510.

(23) Paliwal, R.; Palakurthi, S. Zein in controlled drug delivery and tissue engineering. J. Controlled Release 2014, 189, 108-122.

(24) Patel, A. R.; Heussen, P. C. M.; Dorst, E.; Hazekamp, J.; Velikov, K. P. Colloidal Approach to Prepare Colour Blends from Colourants with Different Solubility Profiles. Food Chem. 2013, 141, $1466-1471$

(25) Rondinelli, J. M.; Kioupakis, E. Predicting and Designing Optical Properties of Inorganic Materials. Annu. Rev. Mater. Res. 2015, 45, 491-518.

(26) Etrich, C.; Fahr, S.; Hedayati, M.; Faupel, F.; Elbahri, M.; Rockstuhl, C. Effective Optical Properties of Plasmonic Nanocomposites. Materials 2014, 7, 727-741.

(27) Kratochvílová, I.; Kovalenko, A.; Fendrych, F.; Petráková, V.; Zálišs, S.; Nesládek, M. Tuning of Nanodiamond Particles' Optical Properties by Structural Defects and Surface Modifications: DFT Modelling. J. Mater. Chem. 2011, 21, 18248.

(28) Johnson, D. I.; Gadd, G. E.; Town, G. E. Total Differential Optical Properties of Polymer Nanocomposite Materials. Proceedings 2006 International Conference on Nanoscience and Nanotechnology, ICONN 2006; pp 423-426.

(29) Rastar, A.; Yazdanshenas, M. E.; Rashidi, A.; Bidoki, S. M. Estimation and Prediction of Optical Properties of PA6/TiO2 Nanocomposites. Arabian J. Chem. 2017, 10, S219-S224.

(30) McClements, D. J. Theoretical Prediction of Emulsion Color. Adv. Colloid Interface Sci. 2002, 97, 63-89.
(31) Kerker, M.; Scheiner, P.; Cooke, D. D.; Kratohvil, J. P. Absorption Index and Color of Colloidal Hematite. J. Colloid Interface Sci. 1979, 71, 176-187.

(32) Wiersma, D. S.; Rivas, J. G.; Bartolini, P.; Lagendijk, A.; Righini, R. Localization or Classical Diffusion of Light? Nature 1999, 398, 207.

(33) Reufer, M.; Rojas-Ochoa, L. F.; Eiden, S.; Sáenz, J. J.; Scheffold, F. Transport of Light in Amorphous Photonic Materials. Appl. Phys. Lett. 2007, 91, 171904.

(34) Schertel, L.; Wimmer, I.; Besirske, P.; Aegerter, C. M.; Maret, G.; Polarz, S.; Aubry, G. J. Tunable High-Index Photonic Glasses. Phys. Rev. Mater. 2019, 3, 015203.

(35) Graves, S. M.; Mason, T. G. Transmission of Visible and Ultraviolet Light through Charge-Stabilized Nanoemulsions. J. Phys. Chem. C 2008, 112, 12669-12676.

(36) Vos, W. L.; Tukker, T. W.; Mosk, A. P.; Lagendijk, A.; IJzerman, W. L. Broadband Mean Free Path of Diffuse Light in Polydisperse Ensembles of Scatterers for White Light-Emitting Diode Lighting. Appl. Opt. 2013, 52, 2602.

(37) Kohl, M.; Lindauer, U.; Royl, G.; Kühl, M.; Gold, L.; Villringer, A.; Dirnagl, U. Physical Model for the Spectroscopic Analysis of Cortical Intrinsic Optical Signals. Phys. Med. Biol. 2000, 45, 37493764.

(38) Zhang, C.; Reufer, M.; Gaudino, D.; Scheffold, F. Improved Diffusing Wave Spectroscopy Based on the Automatized Determination of the Optical Transport and Absorption Mean Free Path. Korea Aust. Rheol. J. 2017, 29, 241-247.

(39) Leung, V. Y. F.; Lagendijk, A.; Tukker, T. W.; Mosk, A. P.; IJzerman, W. L.; Vos, W. L. Interplay between Multiple Scattering, Emission, and Absorption of Light in the Phosphor of a White LightEmitting Diode. Opt. Express 2014, 22, 8190.

(40) Shahabi, S.; Treccani, L.; Rezwan, K. Amino Acid-Catalyzed Seed Regrowth Synthesis of Photostable High Fluorescent Silica Nanoparticles with Tunable Sizes for Intracellular Studies. J. Nanopart. Res. 2015, 17, 270.

(41) de Boer, F. Y.; Kok, R. N. U.; Imhof, A.; Velikov, K. P. White Zein Colloidal Particles: Synthesis and Characterization of Their Optical Properties on the Single Particle Level and in Concentrated Suspensions. Soft Matter 2018, 14, 2870-2878.

(42) Zou, T.; Li, Z.; Percival, S. S.; Bonard, S.; Gu, L. Fabrication, Characterization, and Cytotoxicity Evaluation of Cranberry Procyanidins-Zein Nanoparticles. Food Hydrocolloids 2012, 27, 293-300.

(43) Bilenler, T.; Gokbulut, I.; Sislioglu, K.; Karabulut, I. Antioxidant and Antimicrobial Properties of Thyme Essential Oil Encapsulated in Zein Particles. Flavour Fragrance J. 2015, 30, 392-398.

(44) Ojeda-Mendoza, G. J.; Contreras-Tello, H.; Rojas-Ochoa, L. F. Refractive Index Matching of Large Polydisperse Silica Spheres in Aqueous Suspensions. Colloids Surf., A 2018, 538, 320-326.

(45) De Kruif, C. G.; Briels, W. J.; May, R. P.; Vrij, A. Hard-Sphere Colloidal Silica Dispersions. The Structure Factor Determined with SANS. Langmuir 1988, 4, 668-676.

(46) Rojas, L. F.; Vavrin, R.; Urban, C.; Kohlbrecher, J.; Stradner, A.; Scheffold, F.; Schurtenberger, P. Particle Dynamics in Concentrated Colloidal Suspensions. Faraday Discuss. 2003, 123, 385400.

(47) Vera, M. U.; Durian, D. J. Angular Distribution of Diffusely Transmitted Light. Phys. Rev. E: Stat. Phys., Plasmas, Fluids, Relat. Interdiscip. Top. 1996, 53, 3215-3224.

(48) Laven, P. MiePlot software.

(49) van Blaaderen, A.; Vrij, A. Synthesis and Characterization of Colloidal Dispersions of Fluorescent, Monodisperse Silica Spheres. Langmuir 1992, 8, 2921-2931.

(50) Rivas, J. G.; Sprik, R.; Soukoulis, C. M.; Busch, K.; Lagendijk, A. Optical Transmission through Strong Scattering and Highly Polydisperse Media. Europhys. Lett. 1999, 48, 22-28.

(51) Gómez Rivas, J. Light in Strongly Scattering Semiconductors Diffuse Transport and Anderson Localization. Ph.D. Thesis, Universiteit van Amsterdam, 2002. 
(52) Newton, D. W.; Breen, P. J.; Brown, D. E.; Mackie, J. F.; Kluza,

R. B. Physicochemical Characteristics of Patent Blue Violet Dye. J. Pharm. Sci. 1981, 70, 122-127.

(53) Idouhar, M.; Tazerouti, A. Spectrophotometric Determination of Cationic Surfactants Using Patent Blue V: Application to the Wastewater Industry in Algiers. J. Surfactants Deterg. 2008, 11, 263267.

(54) Jacquemin, D.; Perpète, E. A.; Ciofini, I.; Adamo, C. Accurate Simulation of Optical Properties in Dyes. Acc. Chem. Res. 2009, 42, 326-334. 